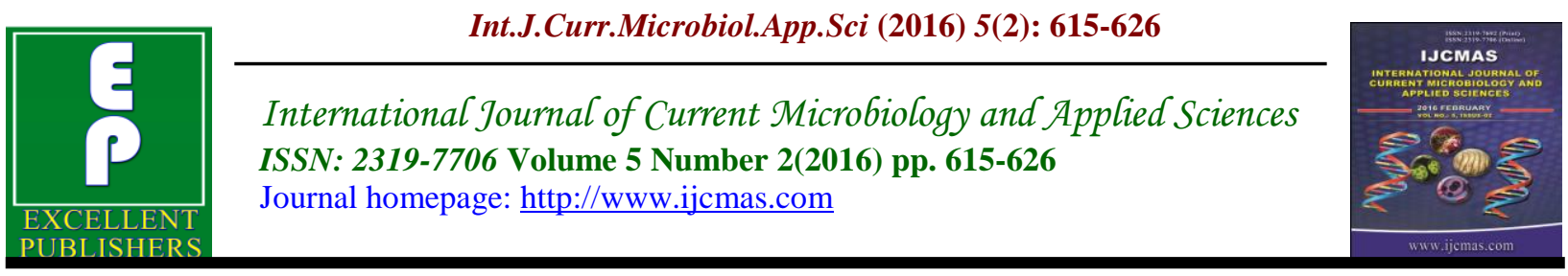

Review Article

doi: http://dx.doi.org/10.20546/ijcmas.2016.502.069

\title{
Neglected Tropical Diseases: Current Snapshot
}

\author{
Priyanka Soni Gupta ${ }^{1} *$, Monica Soni ${ }^{2}$ and Ankur Gupta ${ }^{3}$
}

${ }^{1}$ Department of Microbiology, Jawahar Lal Nehru Medical College, Ajmer, Rajasthan, India

${ }^{2}$ Department of Gynaecology and Obstetrics, P.B.M. Hospital, Bikaner, India

${ }^{3}$ Department of Respiratory Medicine, J.L.N. Medical College, Ajmer, India

*Corresponding author

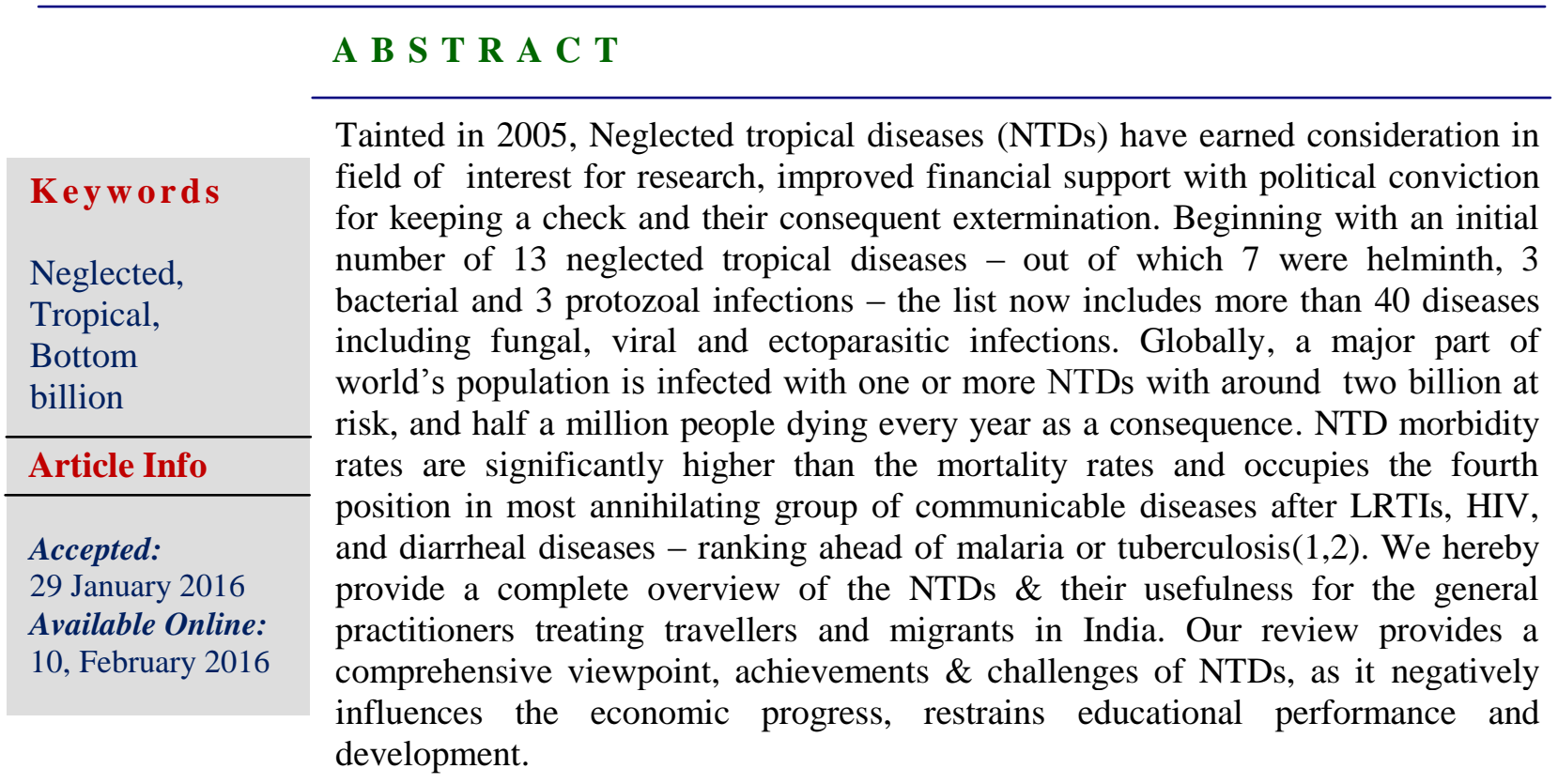

\section{Introduction}

The first publication on "neglected tropical diseases", was written by Molyneux, Hotez \& Fenwick, in November 2005 in PLoS Medicine (3), then came another breakthrough paper in the same journal by Hotez and colleagues (4). These authors reviewed the policy of regular, drug-based intervention for control of NTDs, and planned how this should be combined with the management of the "big three". The proposed strategy was then examined for its strengths and limitations by experts. New England Journal of Medicine subsequently published a review by Hotez et al entitled "Neglected tropical diseases", which clearly indicated that the term "neglected tropical diseases" was gaining popularity (5). The inaugural issue of a new open-access journal, PLoS Neglected Tropical Diseases was published by the Public Library of Sciences, in October 2007(6). The First Report on NTDs by WHO in 2010 brought them to the limelight. Then followed the second report on NTDs by WHO \& London Declaration in 2012, which proposed the control, eradication \& elimination of ten 
NTDs by 2020 ( Soil-transmitted helminths, lymphatic filariasis, Chagas disease, onchocerciasis, leprosy, schistosomiasis, guinea worm, trachoma, visceral leishmaniasis, and human African trypanosomiasis) (7).

\section{Neglected Tropical Diseases}

The World Health Organization highlights 18 NTDs affecting the world's bottom billion of the poorest people living in the tropical zone, and posing a serious health and financial hurdle to developing countries (scabies has been the addition to the list in June' 2014, making the count to 18).(8)

NTDs are assembled because of their chronic, debilitating, and stigmatizing impact on the world's poorest billion, living in remote areas of tropical and subtropical countries $(9,10,11,12-14)$, politically marginalized populations; neglected by research; and subject to control using effective interventions.(15) NTDs have recently gained global attention from all over the world, triggered by burgeoning recognition of NTDs potential hazard to the accomplishment of the Millennium Development Goals by 2015(16).

\section{Evolution of the Neglected Tropical Diseases}

In 2005 and 2006, two influential papers were published, which yielded a lineup of 15 NTDs, 13 out of which were believed to be of particular concern in view of significant morbidity and/or mortality and the global burden $(17,9)$. The initial purview of PLoS Neglected Tropical Diseases was established with this list of 15 diseases which included nine helminth infections (drancunculiasis (guinea worm), lymphatic filariasis, foodborne trematodiasis,, cysticercosis/taeniasis, onchocerciasis and the three main soil- transmitted helminthiases (ascariasis hookworm infection, and trichuriasis)), three protozoal infections (leishmaniasis, Chagas disease and human African trypanosomiasis) and three bacterial infections (Trachoma, Buruli ulcer and leprosy).

The World Health Organization highlighted few more diseases in their list of NTDs, namely dengue/dengue haemorrhagic fever, cholera/epidemic diarrhoeal disease and endemic treponematoses (e.g., pinta, yaws and syphilis) $(18,19)$. At present, the list of NTDs has been widely expanded including more than 40 diseases, which is echoed in the heightened scope of PLoS Neglected Tropical Diseases (20).

\section{Global Snapshot}

NTDs, which span the globe, are endemic to almost 150 countries.(21) Although, the bulk of the NTD load is focussed in lowand middle-income countries in Africa, Latin America and Asia. (22).

\section{General Facts about NTDs (CDC)(23)}

- A minimum of 5 NTDs, simultaneously affects $100 \%$ of lowincome countries.

- Globally, 149 countries \& territories are afflicted by a minimum of 1 neglected tropical disease.

- $\quad$ Premature disability and death is the primary cause of neglected tropical diseases taking a toll on approximately 534,000 people globally every year.

- $\quad$ Most of the NTD mass drug treatment programs costs less than US fifty cents / person /year. 


\section{Guinea Worm Disease}

The Guinea Worm Eradication Program, has helped reduce the number of annual cases of Guinea worm infection from approximately 3.5 million in 20 countries in 1986 to 126 cases in 2014, accounting to a decrease of more than $99 \%$. Only four countries reported the occurrence of Guinea worm disease in 2014 - Ethiopia, Chad, South Sudan and Mali.

\section{Lymphatic filariasis (LF)}

- The Global Programme for Lymphatic Filariasis elimination has called for its elimination by 2020

- People's Republic of China was reported to be the first endemic country certified by WHO, in August 2007, to have successful elimination of lymphatic filariasis, followed by the Republic of Korea in March 2008

- There has been successful interruption of LF transmission in Costa Rica, Trinidad, Suriname and Tobago.

- 1.9 billion treatments for LF were delivered, to more than 570 million people in 48 countries, within the first eight years of the worldwide elimination program.

- Infection of LF to 6.6 million newborns have been avoided.

- The first seven years of the program produced an economic benefit of US $\$ 24$ billion which is expected to exceed US $\$ 55$ billion.

\section{Lymphatic filariasis (LF)}

- The Global Programme for Lymphatic Filariasis elimination has called for its elimination by 2020

- People's Republic of China was reported to be the first endemic country certified by WHO, in August 2007, to have successful elimination of lymphatic filariasis, followed by the Republic of Korea in March 2008

- There has been successful interruption of LF transmission in Costa Rica, Trinidad, Suriname and Tobago.

- 1.9 billion treatments for LF were delivered, to more than 570 million people in 48 countries, within the first eight years of the worldwide elimination program.

- Infection of LF to 6.6 million newborns have been avoided.

- The first seven years of the program produced an economic benefit of US $\$ 24$ billion which is expected to exceed US \$55 billion.

\section{Onchocerciasis}

- Ten West African countries has eliminated the public health problem of Onchocerciasis, and the debilitating blindness it causes.

- In 11 of the 13 major areas of America, Onchocerciasis has been eliminated, where the infection was being transmitted.

\section{Schistosomiasis}

- Schistosomiasis risks around 700 million people.

- Out of which 207 million people are afflicted with the disease.

- About $90 \%$ of schistosomiasis infected people live in Africa.

- The number of people who have been successfully treated for schistosomiasis in Africa have doubled from 2006 to 2009.

- Schistosomiasis treatment is presently ongoing for 27 million school children in Africa. 


\section{Trachoma}

- 57 countries have been reported endemic for trachoma, with about 40 million people being in need of treatment.

- Countries like Iran, Morocco, Mexico, Oman and the United States have eliminated the Blinding trachoma; Several other countries are expected to successfully eliminate this devastating disease soon.

\section{NTDs a Big Bang for Millenium Development Goals}

NTDs have recently reaped immense international attention \& support from the global donors, triggered by increased recognition of their likely threat to the accomplishment of the Millennium Development Goals by 2015.

The Millennium Summit of the UN in 2000, established the Millennium Development Goals (MDGs), which aimed at lifting the world's poorest countries out of poverty by 2015. Out of the 8 listed objectives, MDG 6 was to Combat HIV/AIDS, malaria, and other diseases, applies to NTDs, which fall into the "other diseases" category. Though the concept of NTDs falling under the "other diseases" category is now not so significant, as NTDs have gained much more global attention over the past decade as a powerful progress impediment(16)

The Global Network for NTDs recently released a report summarizing the role played by NTDs in impeding progress in achievement of several MDGs (24). In reference to MDG 4 which aims at Reducing Childhood Mortality, a child infected with any of the helminth parasites will have a compromised immune system with anemic and malnourished condition, making the child much more prone other infections.
Secondly, children harboring one or more NTDs cannot be expected to have regular attendance in school, hindering MDG 2 which aims at Achieving Universal Primary Education.

\section{Global Goals}

With the increasing global attention being focused on NTDs, expanded global efforts are being pursued over the past decade. Significant global NTD goals have been ingrained to be achieved -

The 2012 WHO Roadmap for Implementation, which summarized the objectives \& blueprints for global efforts for NTD control, elimination, and eradication from 2012 to 2020. Goals established were dracunculiasis (Guinea worm disease) eradication by 2015 and yaws by 2020 with elimination of four NTDs -human African trypanosomiasis (sleeping sickness), blinding trachoma, leprosy and lymphatic filariasis- by 2020(25).

\section{The London Declaration on Neglected Tropical Diseases}

Major public and private stakeholders endorsed the London declaration in 2012 and outlined global NTD targets (affirming the goals of Roadmap) and commitments. It main objective was to improve coordination and collaboration in the partner efforts across the respective fields so as to contribute in Guinea worm eradication and by 2020, help elimination of the four NTDs which are blinding trachoma, sleeping sickness, leprosy and LF with control of schistosomiasis, Chagas disease, visceral leishmaniasis, soil-transmitted helminths and onchocerciasis(7).

Global Network for NTDs in collaboration with recent guidelines for drug administration issued by the WHO, are 
integrating their activities in order to project a more unified assault on these conditions. WHO's Strategic and Technical Advisory Group for Neglected Tropical Diseases (STAG-NTD), having appraised the Cochrane review on soil-transmitted parasitic infections published in July 2015, together with the existing body of evidence (some of which was not taken into account in the Cochrane review), notes that research has shown the benefits of deworming children with soil-transmitted helminths. The STAG-NTD therefore continues to endorse WHO's recommendation for mass deworming in areas with prevalence of soiltransmitted helminth infections over $20 \% .(24$

\section{Global Programs on Ntds (8)}

\section{Global Control Efforts}

- Global Programme for Lymphatic Filariasis Elimination

- African Programme for control of Onchocerciasis

- Program for Elimination of Onchocerciasis for America

- Global Efforts for Schistosomiasis Control

- Global Efforts for Control of Soiltransmitted Helminths (STH)

- Global Efforts for Trachoma Control

\section{Global Eradication Efforts}

- Guinea Worm Disease

United States Government Initiatives to Address NTDs
- USAID NTD Program

- Global Health Initiative

Global Programme for Lymphatic Filariasis Elimination

Started in 2000, with a target elimination by 2020 , it aims to interrupt the spread of transmission and minimize the intensity of the disease through administration of anti parasitic drugs.

\section{African Programme for Control of Onchocerciasis}

Launched in 1995, it has reduced the river blindness rates by $73 \%$. Program's approach for treatment of individuals is administration of ivermectin (donated by Merck \& Co., Inc) within the community. Locally distributing the drug has been a significantly effective measure of reducing the disease burden in affected areas.

\section{Program for Elimination of Onchocerciasis for America}

13 areas of six countries in the Americas where the disease still exists, a regional initiative was created in 1992. Reducing blindness and interruption of transmission of onchocerciasis in these 6 countries i.e. Brazil, Colombia, Ecuador, Guatemala, Mexico and Venezuela was the main purpose of this initiative. Ivermectin mass drug administration (MDA) twice per year is the strategy employed to support high treatment coverage, which led to the elimination of blindness in all 13 areas, eight of the 13 areas have witnessed interruption of transmission, and suspected interruptionis expected in two additional areas. The year 2017 has been set as the current target date for elimination of onchocerciasis in the Americas. 


\section{Global Efforts for Schistosomiasis Control}

The drug praziquantel (partially donated by Merck $\mathrm{KGaA}$ ) is given as a yearly dose either to the entire community or to schoolaged children, depending on the disease prevalence.

\section{Global Efforts for Control of Soil- transmitted Helminths (STH)}

In 2001, a resolution was adopted by the World Health Organization which targeted "deworming" with administration of albendazole or mebendazole (donated by Johnson \& Johnson) to $75 \%$ of all at-risk school-age children by 2010 . This is has been the largest public health program attempted till date $\&$ is currently underway.

\section{Global Efforts for Trachoma Control}

In rural Africa and other parts of the developing world, SAFE - a public health strategy approved by the WHO, is underway to treat trachoma epidemics. SAFE is the combination of surgery (S), antibiotics (A), facial cleanliness (F) and environmental educational efforts (E). It is a multifaceted approach for disease control, which has shown favorable results.

\section{Guinea Worm Eradication Program}

In 1980, CDC launched a global campaign for eradication of Guinea worm. In 1986, 20 countries were reported to have GWD, with an incidence of about 3.5 million cases / year \& maximum of $90 \%$ cases from Africa alone. An initiative was taken by The Carter Center, for the global Guinea Worm Eradication Program (GWEP), working in coordination with the ministries of health and developing local, national, and international partnerships.

\section{United States Government Initiatives to} Address NTDs

Over the past ten years, a significant rise in terms of increased U.S. attention and financial backing for NTDs has been observed. In 2006, NTD control Program was launched by the integrated efforts of U.S. Agency for International Development (USAID). In 2008, NTD efforts expansion was announced by the US Government, building on USAID's NTD Program(26). Year 2012, witnessed US Government's approval for the London Declaration.(7)

U.S. NTD efforts are dominantly implemented by USAID. A lot of other agencies including $\mathrm{CDC}, \mathrm{NIH}, \mathrm{DoD}$ and the U.S. FDA have a significant role to play in countering NTDs worldwide.

\section{USAID's NTD Program. ${ }^{(27)}$}

Seven "tool-ready" NTDs, that are specifically responsive to control amidst the availability of effective drugs against them have been targeted by the USAID's NTD Program. 25 countries, largely in subSaharan Africa and Southeast Asia have been covered under this program. The rapidimpact package measure has been used for the USG's support to endemic countries in scaling up MDA and establishing their skills for NTD control programs. The target has been set for onchocerciasis elimination in the Americas by 2016 and elimination of blinding trachoma \& LF globally by 2020 .

An expanded approach to USG-supported NTD control and elimination efforts have been announced by President Obama in May 2009, in form of United States Global Health Initiative (GHI).

Other USG agencies have also been active in supporting research and development activities pertinent to approval of control 
tools for NTDs. The 7 targeted NTDs along with those not considered "tool-ready" (Buruli ulcer, Chagas disease, dengue, human African trypanosomiasis, leishmaniasis) are being considered primarily (29). NTD efforts have been supported by various other organizations like $\mathrm{NIH}, \mathrm{CDC}$, and $\operatorname{DoD}(30)$. A voucher is awarded by FDA during the approval of certain drugs preventing or treating an NTD, which can be redeemed for a priority review of a drug application for any indication, submitted later (31). It has been proposed as an incentive to the private sector for promoting their investments in new NTD drug development(32). Technical assistance is also provided by $\mathrm{CDC}$ to the countries and other partners, in developing protocol for control of NTDs and efforts for monitoring and evaluating progress(33).

\section{NTDS : Progress and Challenges}

NTDs were brought to the forefront by the first WHO report in 2010. The second WHO report (2012) closely monitored the progress achieved with these interventions along with the obstacles to their operation. And then, closely followed the 2012 London Declaration which targeted the control, eradication or elimination by 2020 of the 10 NTDs (lymphatic filariasis, trachoma, soiltransmitted helminths, onchocerciasis, schistosomiasis, leprosy, guinea worm, visceral leishmaniasis, Chagas disease and human African trypanosomiasis). In 2015, the third WHO report, for the first time analyses the wise investments that are required for the achievement of the targets set by WHO Roadmap on NTDs along with the universal coverage for NTDs. Some successful achievements from the time of the London Declaration, have motivated for the possibility of control and eradication of NTDs. Colombia has been recently reported to be the world's first country to accomplish onchocerciasis eradication. Guinea worm has been recently eradicated from Nigeria, Niger and the Ivory Coast. 23 countries have treated a minimum of $75 \%$ of children who were at risk for soil-transmitted helminth infections. Blinding trachoma has cut the unmapped regions of the world for this disease in half with the massive disease mapping program using mobile technology.

Of all the drugs approved for distribution, only $60-70 \%$ are reaching the people in need of them (34).

Mass drug administration program's implementation and research must be a priority. Political unrest, natural disasters or even epidemics like the Ebola outbreak can quickly wreck an ongoing control program.

Innovative research for the new validated tools and strategies with the potential to be applied in the field, calls for additional funding but the important lacunae in our knowledge of the epidemiology and NTDs control still remain. The emergence and reemergence of NTDs along with the concerns regarding their brunt on the future human development are some of the new and more challenging issues in the 21st century. Globally, NTDs are complex involving a panel of contributing factors, accelerated by globalization with unique challenges to be presented for the collective \& unified global public health efforts. International community envisioned NTDs to be a scourge on human progress through the MDGs., being a critical hurdle to pacify worldwide poverty.

Inspite of the promises that have been made, a paucity of basic diagnostics tools, vaccines and drugs required to support the suffering populations still exists. An appropriate coordination between the Global public health guidelines and measures that desire to 
face the unique risks presented by NTDs is needed, along with the reciprocation to the multiple factors in association with these diseases, ranging from control of vector to pacifying poverty \& underlining social determinants, facing climate change, interrupting spread via international travel and trade, providing more powerful monitoring and financial support.

Table.1 Seven NTDs Targeted by USAID's NTD Program(28)

Table 1: Seven NTDs Targeted by USAID's NTD Program(28)

\begin{tabular}{|c|c|c|c|}
\hline Disease & $\begin{array}{l}\text { People } \\
\text { Affected Each } \\
\text { Year }\end{array}$ & $\begin{array}{l}\text { Affected } \\
\text { Countries }\end{array}$ & Causes \\
\hline $\begin{array}{l}\text { Soil-Transmitted } \\
\text { Helminths (STH): }\end{array}$ & 1,500 million + & 112 & $\begin{array}{l}\text { Direct exposure to soil contaminated with } \\
\text { human feces containing STH eggs \& } \\
\text { worms being transmitted through ingestion. }\end{array}$ \\
\hline Ascariasis (roundworm) & 819 million & - & $\begin{array}{l}\text { Ingestion of contaminated soil, food, or } \\
\text { water }\end{array}$ \\
\hline Hookworm & 439 million & - & $\begin{array}{l}\text { Barefoot walking on contaminated soil or } \\
\text { ingesting larvae }\end{array}$ \\
\hline Trichuriasis (whipworm) & 465 million & - & $\begin{array}{l}\text { Ingestion of contaminated soil, food, or } \\
\text { water }\end{array}$ \\
\hline Lymphatic filariasis & 120 million & 73 & Worms transmitted by mosquitoes \\
\hline Onchocerciasis & 37 million & 36 & $\begin{array}{l}\text { Worms transmitted by black flies breeding } \\
\text { near fast-moving rivers and streams }\end{array}$ \\
\hline $\begin{array}{l}\text { Schistosomiasis (snail } \\
\text { fever) }\end{array}$ & 249 million* & 78 & $\begin{array}{l}\text { Worms transmitted through contaminated } \\
\text { freshwater inhabited by parasite carrying } \\
\text { snails. }\end{array}$ \\
\hline Trachoma & 29 million+ & 51 & $\begin{array}{l}\text { Direct or indirect contact with bacteria in } \\
\text { an infected person's eye or nasal discharge } \\
\text { (on hands/clothes or on the feet of flies) }\end{array}$ \\
\hline
\end{tabular}

NOTES: - information unavailable; *requiring preventive chemotherapy; + active trachoma, trichiasis, and irreversible blindness due to trachoma.

A rapid pace in securing the global control of NTDs call for an active role to be played by the newly developed blueprints that focus upon integration, such as the One Health concept, multiple stakeholder cooperation through public-private partnerships. To help the "bottom billion" out of the NTDs trap \& interrupting their transmission to other areas, persistent policy advocacy with commitment, financing and exploration of 
these policies have a crucial role to play. Its only through these interventions that the global health community can hope to pacify the extreme suffering \& morbidity produced by NTDs, working in coordination for their assured elimination and eradication for the present and the future generations.

The global elimination of blinding trachoma, HAT, LF, onchocerciasis, trachoma and leprosy as public health problems can be expected within the next decade with continued efforts being put in, along with eradication of dracunculiasis. New drugs and vaccines may be called for some of the high prevalence NTDs like hookworm infection, schistosomiasis, Chagas disease and leishmaniasis. Because of their potential for improving health \& economic development, NTDs vaccines are sometimes referred to as antipoverty vaccines $(37,38)$. NTD vaccines may take a decade or more for their widespread global acceptance, as at present they are entering early stage clinical trials.

Much can be gained by extermination of these diseases. With the global efforts focussed on decreasing the load of NTDs among the world's bottom billion, key issues for USG NTD efforts success targets : maintaining and strengthening achievements, such as increased scope of economic and adequate measures for NTDs control (39); realizing further cost efficiency savings; procuring donated drugs; enhancing country's strength for conducting intense case detection and management; support for enhanced surveillance and assessment of global momentum; supporting eminent research trials with identification of costeffective measures for not yet "tool-ready" NTDs; further collaborating USG global health programs with other NTD efforts for achieving desired outcome.
With the clock running out on the achievement of 2015 Millennium Development Goals, outlining strategy for the post-2015 feasible Development Goals is well in progress.

\section{References}

1. WHO, Investing to Overcome the Global Impact of NTDs, 2015.

2. WHO, Neglected Tropical Diseases: Hidden Successes, Emerging Opportunities, 2009; USAID's NTD Program website, www.neglecteddiseases.gov.

3. Molyneux DH, Hotez PJ, Fenwick A. "Rapid-impact interventions": how a policy of integrated control for Africa's neglected tropical dis-eases could benefit the poor. PLoS Med. 2005;2:e336.

4. Hotez PJ, Molyneux DH, Fenwick A, Ottesen E, Ehrlich Sachs S, Sachs JD. Incorporating a rapid-impact package for neglected tropical dis-eases with programs for HIV/AIDS, tuberculosis, and malaria. PLoS Med. 2006;3:e102.

5. Hotez PJ, Molyneux DH, Fenwick A, Kumaresan J, Ehrlich Sachs S, Sachs JD, et al. Control of neglected tropical diseases. N Engl J Med. 2007;357:1018-27.

6. Hotez P. A new voice for the poor. PLoS Negl Trop Dis. 2007;1:e77

7. The London Declaration on Neglected Tropical Diseases, Jan. 30, 2012.

8. WHO Pages on Neglected Tropical Diseases and Others: http://www.who.int/neglected_d iseases/diseases/en/; http://www.who.int /neglected_diseases/diseases/others/en/

9. Hotez PJ, Molyneux DH, Fenwick A, Ottesen E, Ehrlich Sachs S, Sachs JD. Incorporating a rapid-impact package for neglected tropical dis-eases with programs for HIV/AIDS, tuberculosis, and malaria. PLoS Med. 2006;3:e102 
10. Utzinger J, Raso G, Brooker S, de Savigny D, Tanner M, Ørnbjerg N, et al. Schistosomiasis and neglected tropical diseases: towards in-tegrated and sustainable control and a word of caution. Parasitology. 2009;136:185974.

11. Hotez PJ, Fenwick A, Savioli L, Molyneux DH. Rescuing the bottom billion through control of neglected tropical diseases. Lancet. 2009;373:1570-5.

12. WHO. Working to overcome the global impact of neglected tropical dis-eases. First WHO report on neglected tropical diseases. Geneva: World Health Organization. 2010.

13. Hotez PJ. The four horsemen of the apocalypse: tropical medicine in the fight against plague, death, famine, and war. Am J Trop Med Hyg. 2012;87:310.

14. Litt E, Baker MC, Molyneux D. Neglected tropical diseases and mental health: a perspective on comorbidity. Trends Parasitol. 2012;28:195-201.

15. WHO, Working to Overcome the Global Impact of NTDs, 2010. The 17 are: Buruli ulcer, Chagas disease, cysticercosis, dengue, dracunculiasis (Guinea worm disease), echinococcosis, foodborne trematode infections, human African trypanosomiasis (sleeping sickness), leishmaniasis, leprosy, lymphatic filariasis, onchocerciasis (river blindness), rabies, schistosomiasis, soil-transmitted helminths, trachoma, and yaws.

16. United Nations, The Millennium Development Goals Report 2008, Sept. 2008; Congressional Research Service (CRS), Neglected Tropical Diseases: Background, Responses, and Issues for Congress, Jan. 2011; Global Network "NTDs and the Millennium Development Goals," fact sheet, undated.

17. Molyneux DH, Hotez PJ, Fenwick A.
"Rapid-impact interventions": how a policy of integrated control for Africa's neglected tropical dis-eases could benefit the poor. PLoS Med. 2005;2:e336.

18. Utzinger J, Raso $\mathrm{G}$, Brooker $\mathrm{S}$, de Savigny D, Tanner M, Ørnbjerg N, et al. Schistosomiasis and neglected tropical diseases: towards in-tegrated and sustainable control and a word of caution. Parasitology. 2009;136:185974.

19. WHO. Neglected tropical diseases: hidden successes, emerging opportunities. Geneva, World Health Organization 2006.

20. Hotez PJ, Yamey G. The evolving scope of PLoS Neglected Tropical Diseases. PLoS Negl Trop Dis. 2009;3:e379.

21. WHO: "Neglected tropical diseases: diseases,"

webpage, http://www.who.int/neglected _diseases/diseases/en/;Neglected

Tropical Diseases: Hidden Successes, Emerging Opportunities, 2009; Investing to Overcome the Global Impact of NTDs, 2015.

22. WHO, Neglected Tropical Diseases: Hidden Successes, Emerging Opportunities, 2009; USAID's NTD Program website, www.neglecteddiseases.gov; $\mathrm{WHO}$, Investing to Overcome the Global Impact of NTDs, 2015

23. CDC, "Neglected Tropical Diseases: CDC's Role," webpage, http://www.cdc.gov/globalhea lth/ntd/cdc_role/index.html.

24. The Global Network for Neglected Tropical Diseases. Neglected Tropical Diseases and the Post-2015 Development Agenda.http://www.globalnetwork.org/s ites/default/files/Neglected\%20Tropical $\% 20$ Diseases $\% 20$ and $\% 20$ the $\% 20$ Post2015\%20Development\%20Agenda\%20 2013.pdf. Accessed Nov 232014.

25. WHO, Accelerating Work to Overcome 
the Global Impact of Neglected Tropical

Diseases: A Roadmap for Implementation, 2012.

26. White House, "President Bush Announces New Global Initiative for Combating Neglected Tropical Diseases," press release, Feb. 20, 2008; USAID's NTD Program website, www.neglecteddiseases.gov; CRS, Neglected Tropical Diseases: Background, Responses, and Issues for Congress, Jan. 2011.

27. USAID, "Neglected Tropical Diseases," webpage, http://www.usaid.gov/whatwe-do/global-health/neglected-tropicaldiseases; USAID's NTD Program website, www.neglecteddiseases.gov.

28. WHO, Neglected Tropical Diseases: Hidden Successes, Emerging Opportunities, 2009; Peter Hotez, et al., "Control of Neglected Tropical Diseases," NEJM, Vol. 357 (10), 2007; WHO fact sheets: "Soil-transmitted helminth infections," April 2014; "Lymphatic filariasis," March 2014; "Schistosomiasis," February 2014; "Onchocerciasis," March 2015; "Blinding Trachoma," March 2015; WHO, The 17 NTDs - Summary webpage,http://www.who.int/neglected_ diseases/diseases/summary/en/; WHO, Investing to Overcome the Global Impact of NTDs, 2015; Rachel Pollan, et al., "Global numbers of infection and disease burden of soil transmitted helminth infections in 2010," Parasites \& Vectors, Vol. 7: 37, 2014.

29. WHO, "NTDs: Innovative and Intensified Disease Management," brochure, 2007.

30. NIH/NIAID, “NIAID's Role in NTD Research,"

webpage,http://www.niaid.nih.gov/topic s/tropicalDiseases/research/Pages/role.a spx; CDC, "Neglected Tropical Diseases: CDC's Role," webpage, http://www.cdc.gov/globalhea lth/ntd/cdc_role/index.html; KFF, The U.S. Department of Defense and Global Health: Infectious Disease Efforts, 2013.

31. FDA: Guidance for Industry: Neglected Tropical Diseases of the Developing World: Developing Drugs for Treatment or Prevention, July 2014; Guidance for Industry: Tropical Disease Priority Review Vouchers (draft), Oct. 2008.

32. Per Jesse L. Goodman, FDA Chief Scientist, "Working Together to Address the Challenges of Rare and Neglected Diseases," Testimony before the House Foreign Affairs Committee's Subcommittee on Africa, Global Health, Global Human Rights, and International Organizations, June 27, 2013, "FDA has long had in place a review system to ensure that the most critical medical products are reviewed on a priority basis. The goal for Priority Review applications for products that offer major advances in treatment, or provide a treatment when no adequate therapy exists, is to complete them within a sixmonth period, compared to the 10month goal for standard review of other products. The Food and Drug Administration Amendments Act of 2007 (FDAAA) granted FDA the authority, beginning in 2009, to award Priority Review vouchers to a company that submits and, after review, receives marketing approval for certain products for one of 16 neglected 'tropical' diseases listed in the legislation. If transferred to apply to a blockbuster drug, the four months of earlier market access available when a Priority Review voucher is redeemed could, in some circumstances, be very valuable."

33. CDC, "Neglected Tropical Diseases: CDC's Role," webpage, http://www.cdc.gov/globalhea lth/ntd/cdc_role/index.html.

34. Uniting to Combat NTDs. Delivering on Promises and Driving Progress: 
Second

Progress

Report. http://unitingtocombatntds.org/r esource/delivering-promises-and-

driving-progress-second-progress-report

35. USAID's NTD Program website, www.neglecteddiseases.gov; USAID, "Neglected Tropical Diseases," fact sheet, Sept. 18, 2012; WHO Regional Office for Africa (AFRO), Regional Strategy on NTDs in the WHO African Region 2014-2020, 2014; AFRO, Regional Strategic Plan for Neglected Tropical Diseases in the African Region 2014-2020, 2013; The END Fund, http://www.end.org/; Emily Toubali, "ENVISION Resumes Support for Mali's NTD Program," April 21, 2014,

NTD

Spotlight,http://www.ntdenvision.org/sp otlight/envision_resumes_support_for_ malis_ntd_program.

36. Through FY 2013. USAID, Annual Progress Report to Congress: Global Health Programs FY 2013, 2014; USAID's NTD Program website, www.neglecteddiseases.gov; USAID, "Neglected Tropical Diseases," fact sheet, Sept. 18, 2012, http://www.usaid.gov/newsinformation/fact-sheets/neglectedtropical-diseases.

37. Hotez PJ, Ferris MT. The antipoverty vaccines. Vaccine 2006;24:5787-99.

38. Hotez P. A handful of 'antipoverty' vaccines exist for neglected diseases, butthe world's poorest billion people need more. Health Affairs 2011;30:1080-7.

39. The Global Network for Neglected
Tropical Diseases. Neglected Tropical Diseases and the Post-2015 Development Agenda.http://www.globalnetwork.org/s ites/default/files/Neglected\%20Tropical $\% 20$ Diseases $\% 20$ and $\% 20$ the $\% 20$ Post2015\%20Development $\% 20$ Agenda\%20 2013.pdf

\section{Links of interest:}

WHO Pages on Neglected Tropical Diseases and Others: http://www.who. int/neglected_diseases/diseases/en/; http ://www.who.int/neglected_diseases/dise ases/others/en/

WHO Accelerating Work to Overcome the Global Impact of Neglected Tropical Diseases: http://www.who.int/neglected _diseases/NTD_RoadMap_2012_Fullve rsion.pdf

66th World Health Assembly Resolution on NTDs: http://www.who.int/neglected_di seases/mediacentre/WHA_66.12_Eng.p df

Uniting to Combat NTDs: The London Declaration: http://unitingtocombatntds. org/resource/london-declaration

The Overseas Development Institute's Post2015

tracker: http://post2015.org; http://tracke r.post2015.org

Bill Gates' keynote address to the American Society of Tropical Medicine and Hygiene: http://tropmed2014.tumblr.co $\mathrm{m} /$ post/102457469758/recorded-onsunday-november-2nd-2014-bill-gates

\section{How to cite this article:}

Priyanka Soni Gupta, Monica Soni and Ankur Gupta. 2016. Neglected Tropical Diseases: Current Snapshot. Int.J.Curr.Microbiol.App.Sci. 5(2): 615-626. doi: http://dx.doi.org/10.20546/ijcmas.2016.502.069 